\title{
Performance Analysis of Low-Cost Infrared Sensors for Multi-Robot Localization and Communication
}

\author{
Abdulmuttalib T. Rashid \\ Electrical Engineering Department \\ University of Basrah \\ Basrah, Iraq
}

\begin{abstract}
In this paper, we produced a study for choosing a suitable environment for indoor localization and communication among several robots by using low-cost infrared sensors. This study is used to compare the performance of the infrared sensors for measuring the distances and the range of communication among robots at indoor environments. This environment is tested under the influence of the sun and the fluorescent lights and with different angles of viewing among these sensors. Practical circuits for these infrared sensor are built and tested in different environments with different angles of view to choose a suitable performance of these sensors.
\end{abstract}

\section{General Terms}

Multi-robot system, Infrared sensors

\section{Keywords}

Infrared sensors, Distance IR sensor, localization, robots communication

\section{INTRODUCTION}

Multi-robot systems have several capabilities in localize themselves more efficiently [1,2], improve the speed of path planning and exploration missions $[3,4]$, accomplish task in shorter times and consist of simple robots that have the ability to avoid the collision with each other [5]. All these capabilities need to equip the robots with sensors which used for different kinds of measurements such as: motion, temperature, light ...etc. [6].

In multi-robot system, sensors maybe used for communication, distance measurements, obstacle detection etc. [7, 8]. Localization represents a great problem for choosing suitable sensors for distance measurement in multirobot system [9]. Some sensors such as: laser scanner, infrared and ultrasonic sensors can be equip on a robot for distance measurement [10-12]. In many applications low cost sensors are used for measuring the distance instead of the expensive sensors like the laser scanner and camera [13-15].

On the other hand, not only the distance calculation is needed for localization; Localization algorithms need to know the identity of sender and receiver and some of them are depended on the connectivity among nodes to estimate the nodes' locations. Again, we are looking for inexpensive sensors to achieve communication among nodes for an indoor system which are the infrared sensors [16].

This paper aims to address the performance of sensors that are concerned with the distance measurement, having a low price and providing accurate calculations to avoid any misunderstanding that may face the industrial people while measuring the distance of objects with different colors. Section 2 describes the sensors used in this paper and all the experiments and comparisons have been done in section 3 . Section 4 includes the conclusion.

\author{
Abduladhem A. Ali \\ Computer Engineering Department \\ University of Basrah \\ Basrah, Iraq
}

\section{SYSTEM DESCRIPTION}

This section includes description two types of sensors that can be used for robots localization. These sensors are the Infrared sensor and the Distance IR sensor.

\section{Infrared sensor}

Infra-red transmitter and receiver sensors are electronic devices that emit and detect infrared radiation in order to sense some aspect of the surroundings, as shown in Fig. 1. These sensors can be grouped as: intensity based infrared, modulated infrared and infrared ranging sensors.

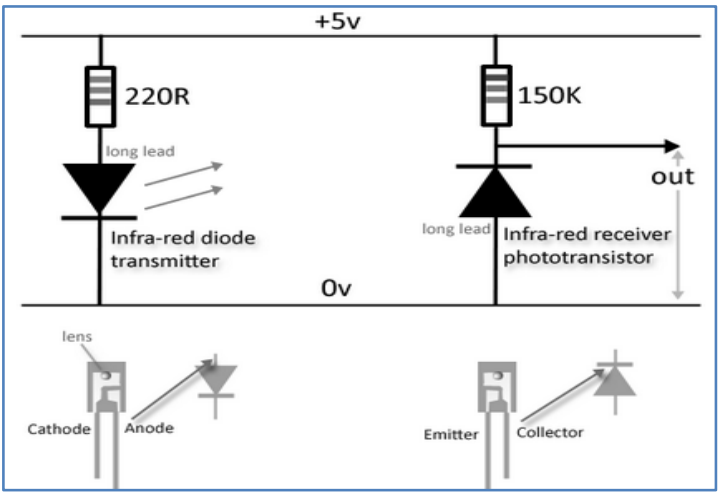

Fig. 1. Infra-red transmitter and receiver sensors.

\section{Distance IR sensor}

Infrared ranging sensors are distance sensors that have IR transmitter, focusing lens and position sensitive detector and they calculate the distance based on triangulation [17]. Fig. 2 shows sharp IR range sensors as examples of these sensors.

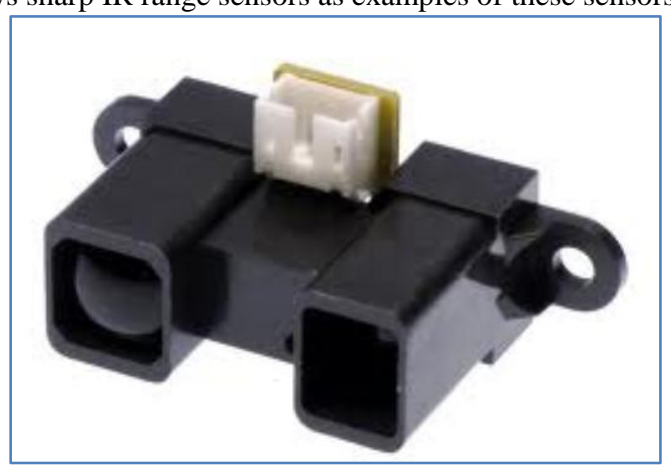

Fig. 2. Sharp 2Y0A21 distance IR sensor [17].

The Sharp IR Range sensor is the most powerful sensor available to the robot applications. It is particularly effective, easy to use, very cheap, very small, good range, and has low power consumption. A major advantage you may have with the Sharp IR rangefinder is beam width. It's properly thin meaning to detect an object which is pointed directly by the 
sensor. The Sharp IR Range sensor uses the process of triangulation. A pulse of light which has wavelength range of $850 \mathrm{~nm}$ is emitted and then reflected back by angle as shown in Fig. 3. Triangulation principle used this reflected beam angle to determine the distance.

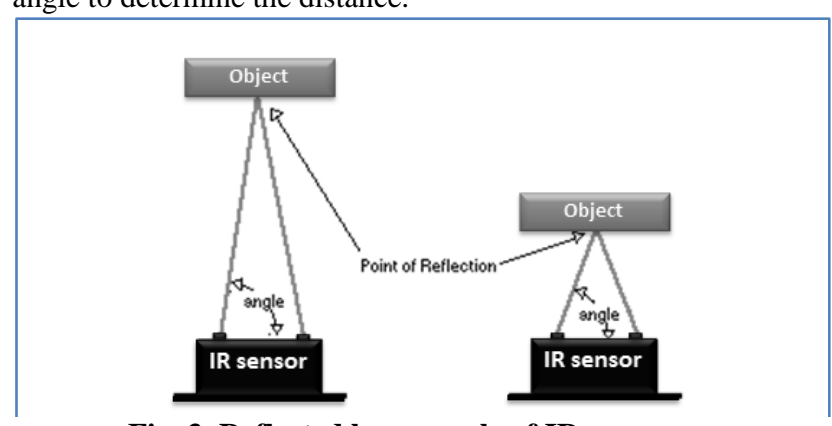

Fig. 3. Reflected beam angle of IR sensor.

The Sharp IR has a non-linear output as shown in Fig. 4. This means that as the distance increases linearly, the analog output voltage increases non-linearly. To effectively use the Sharp IR Range sensor, a lookup table or a representative equation of the distance function can be either implemented. Notice that from Fig. 4 the Sharp IR Range sensor has not proper response at the beginning of the graph. This is because the range sensor is not capable of detecting very short distances. One major drawback with the Sharp IR Range sensor is the minimum sensor range. This is when an object is so close that the sensor cannot get an accurate reading, and it gives erroneous information about the actual position of robot. The solution to this problem is to put the sensor out of this bad range as shown in Fig. 5. Another drawback of these range sensors is the problem of cross interference. This means that the signal emitted by one sensor can possibly be read by another sensor and therefore give bad readings.

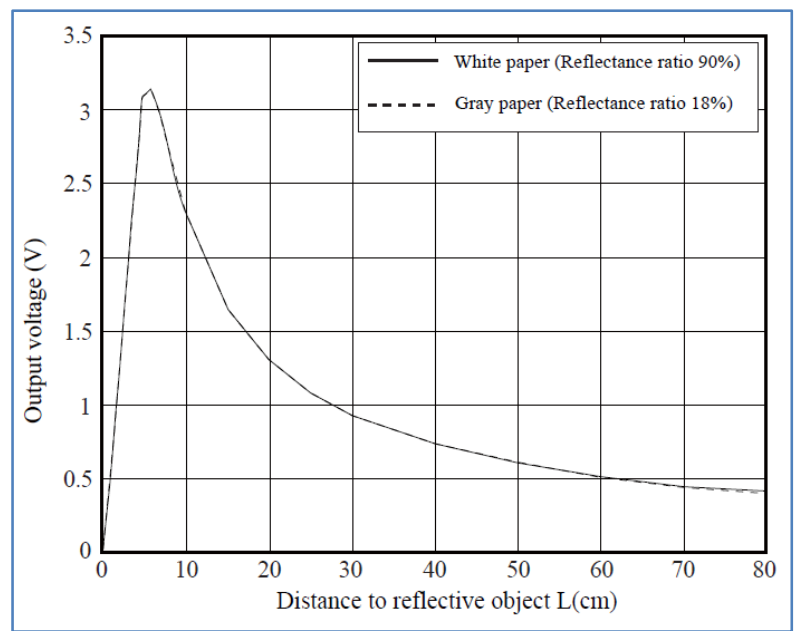

Fig. 4. Distance VS Analog Output voltage [17].

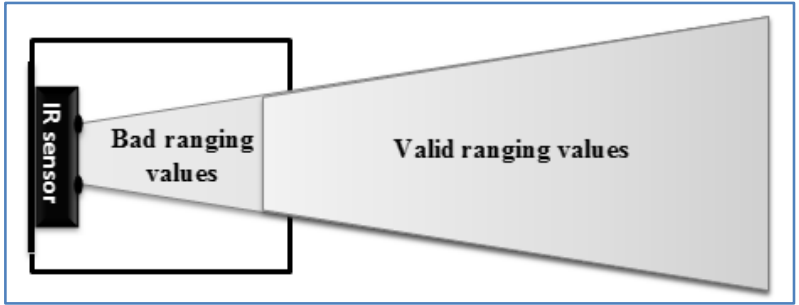

Fig. 5. Valid ranging values for IR sensor.

One of the most uses of the Sharp IR sensor is to do mapping. For this process, a one range sensor, and a one servo motor are needed. The idea is to place the range sensor on the moving servo, then to rotate the servo to some degree, take a distance reading, and record it. After the first cycle, servo must rotate back to the other direction, so the values must be stored in the array in reverse order. This array contains numbers, which corresponds to distances, which correspond to adjacent angles.

\section{EXPERIMENTAL RESULTS}

\section{The distance of communication}

This experiment is used to estimate the distance which grantees the communication between two robots. Fig. 6 shows the device used for this experiment. This device has two circuits of infrared sensors supplied by 5 volts. Each circuit has Infrared transmitter, Infrared receiver and operational amplifier works as a comparator circuit at each IR detector circuit as shown in Fig. 7. The IR intensity at different viewing angles is illustrated in Fig. 8. This experiment demonstrates that the received IR power will decrease when the reference voltage of comparator decreases. Fig. 8 (a) shows the case when each robot equipped with 6 infrared sensors. Data transmission from one robot to another using digital state for input signal, allows acceptable signal range at $23 \mathrm{~cm}$ with $\pm 1 \mathrm{~cm}$ tolerance when reference voltage is 0.7 volts. Fig. 8 (b) shows the case for 12 infrared sensors. Data transmission from one robot to another using digital state for input signal, allows acceptable signal range at $72 \mathrm{~cm}$ with $\pm 1 \mathrm{~cm}$ tolerance when reference voltage is 0.7 volts. 


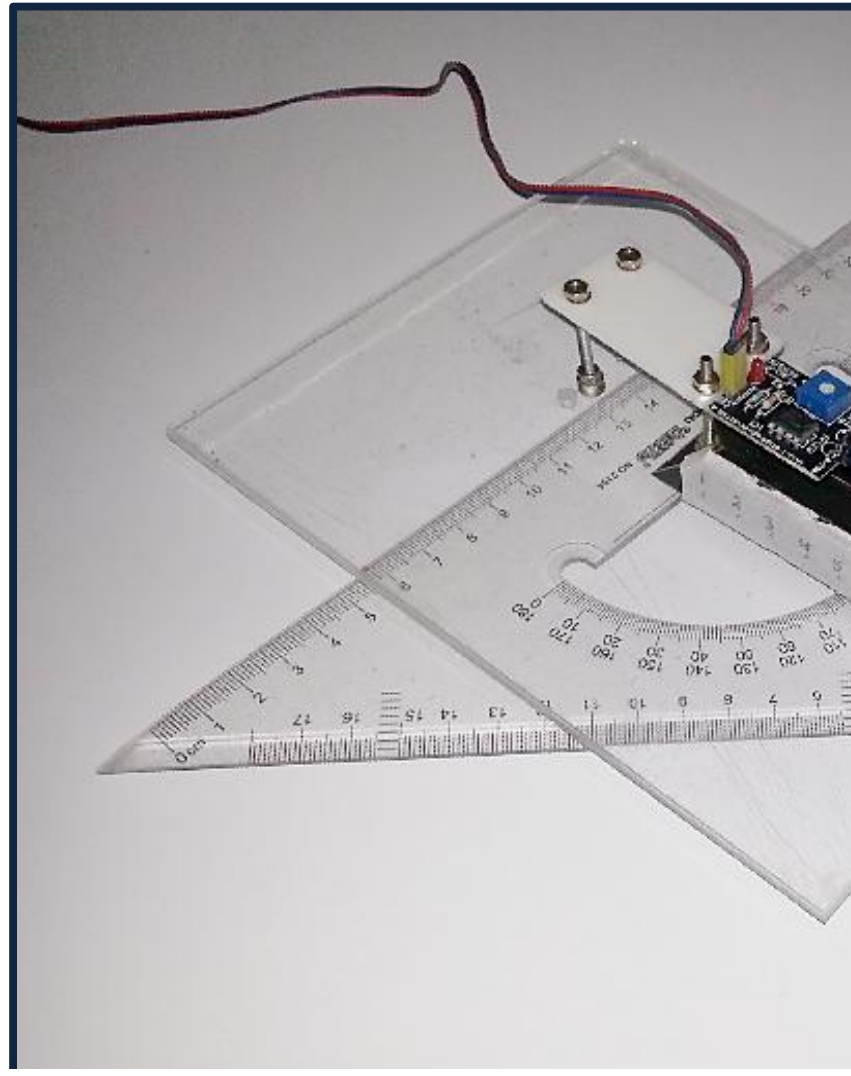

Fig. 6. The experiment of Infrared sensors.

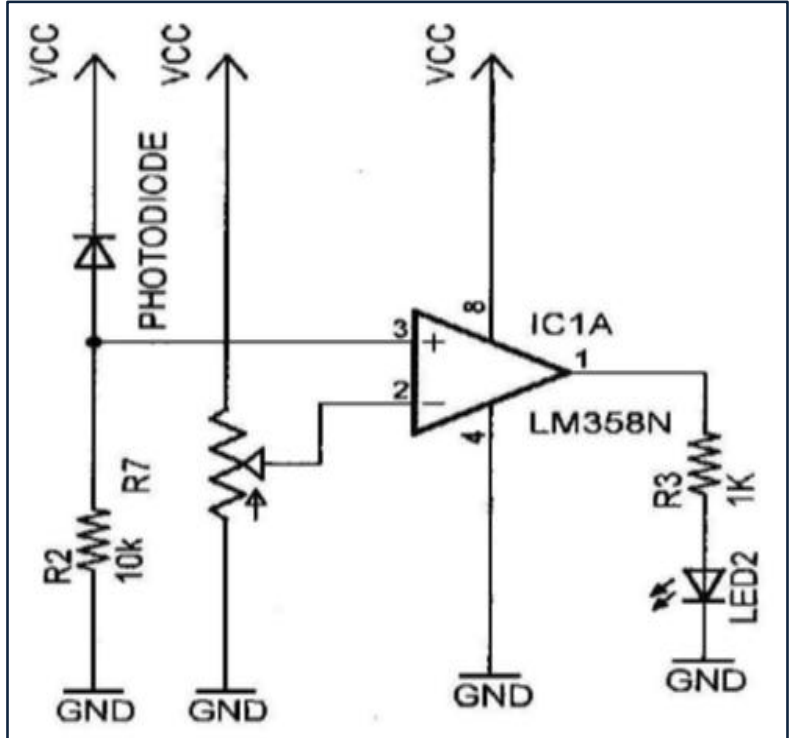

Fig. 7. The infrared sensor circuit.

\section{The distance between two robots}

These experiments are used to measure the distance between two robots in different environments. The distance IR sensor was tested in two lighting environments: sunlight, and fluorescent light that is available in experimental environment. The sunlight includes infrared radiation of about $50 \%$, which could affect the measured value. The fluorescent light room which has a wavelength less than $700 \mathrm{~nm}$ is similar with measurement from dark room. Also the white body and black body obstacles are tested to estimate the distance to infrared sensor. Fig. 9 (a) shows the distance estimation of IR signal reflection with white body robots for both sunlight and fluorescent light room. White body robots reflect more IR radiation in sunlight. The measured samples with black body are shown in Fig. 9 (b). The black body is not a good infrared reflector. As a result the white body robots are more suitable than black body robots, and environment with sunlight is not suitable for experiment because it contains some infrared radiation which influences the measurements. Distance estimation of distance IR sensor would depend on measured IR signals which are reflected from robots. The distance for robot is illustrated as converted ADC values of reflected IR radiation.

The accuracy of the distance IR sensor is tested for different angles of rotating (four, six, and eight degrees) as shown in Fig. 10. This experiment is repeated about 100 times for each distance in order to estimate the median error for each distance. This error occurs due to the circular shape of robot and the point at which distance sensor takes it measurement. The four degrees case has small median error, but it needs a larger time to complete the scanning of the environment.

At large rotation angles the distance IR sensor has less time for complete scanning of environment, but this at first leads to increase the median error, and also the problem of visibility arises. Robot visibility distinguishes if robot is visible by distance IR sensor or not. Fig. 11 shows that the percentage of robot visibility is decreased as the rotation angle is increased and as the distance between robot and distance IR sensor is increased. 


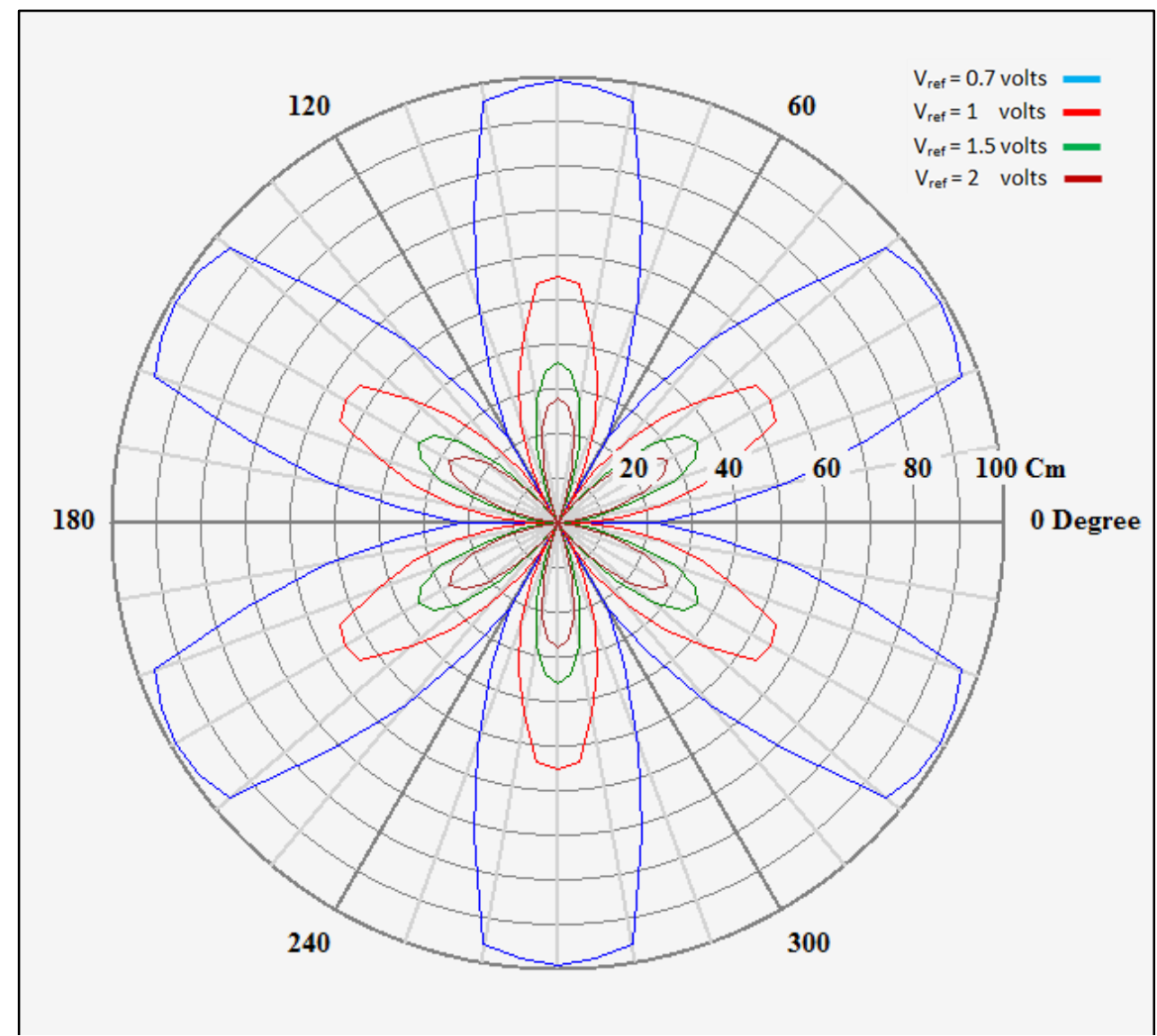

(a)

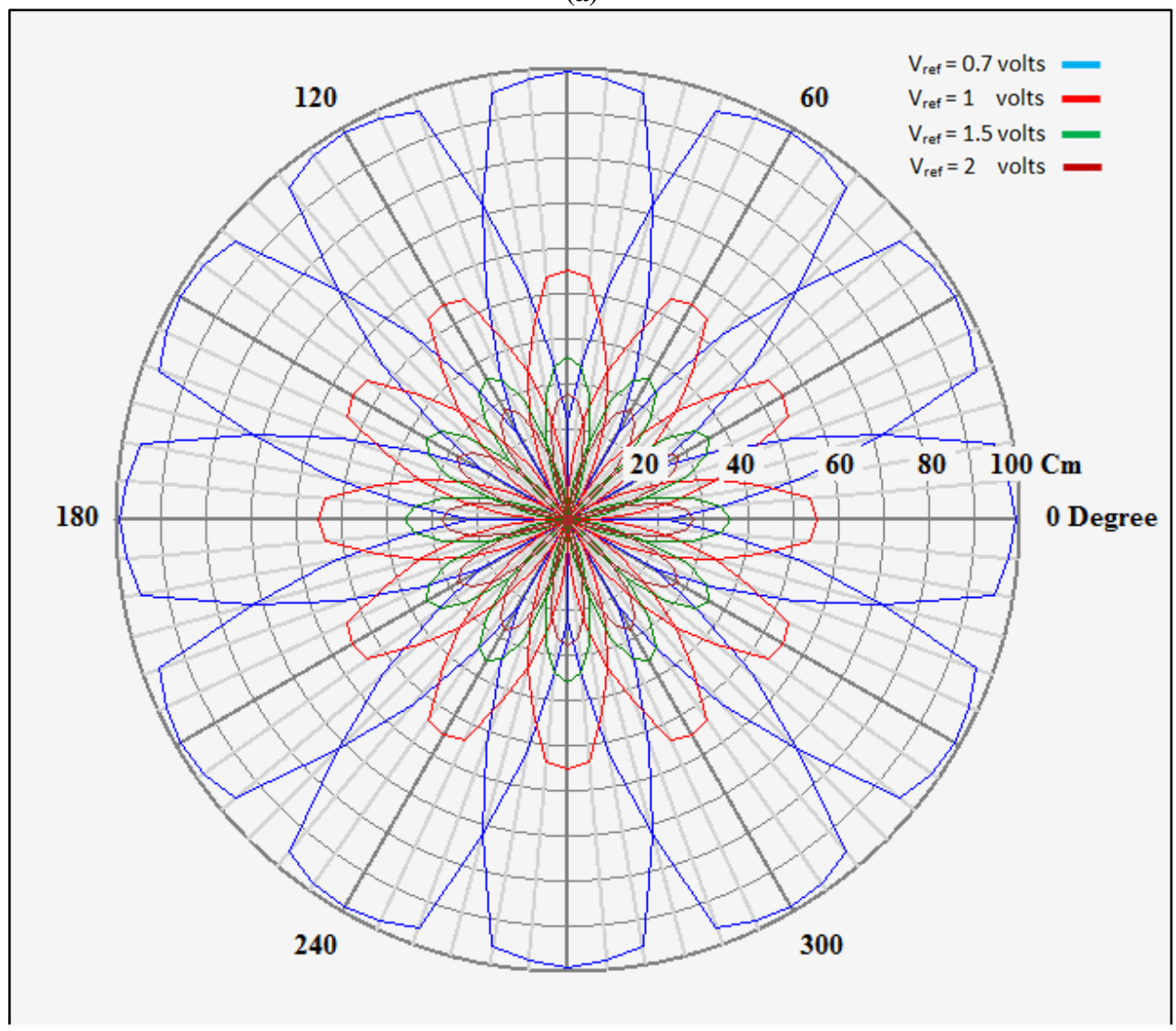

(b)

Fig. 8. The distance estimation of IR received signal between two robots. (a) 6 infrared sensors. (b) 12 infrared sensors. 
(a)

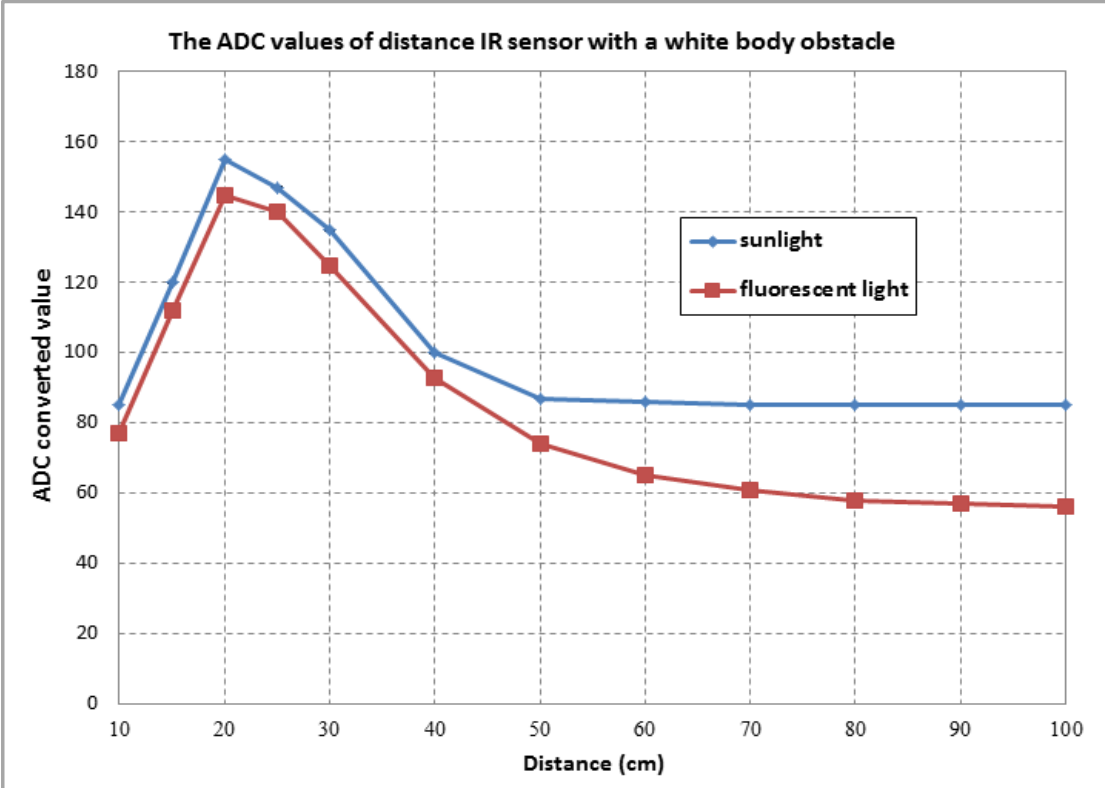

(b)

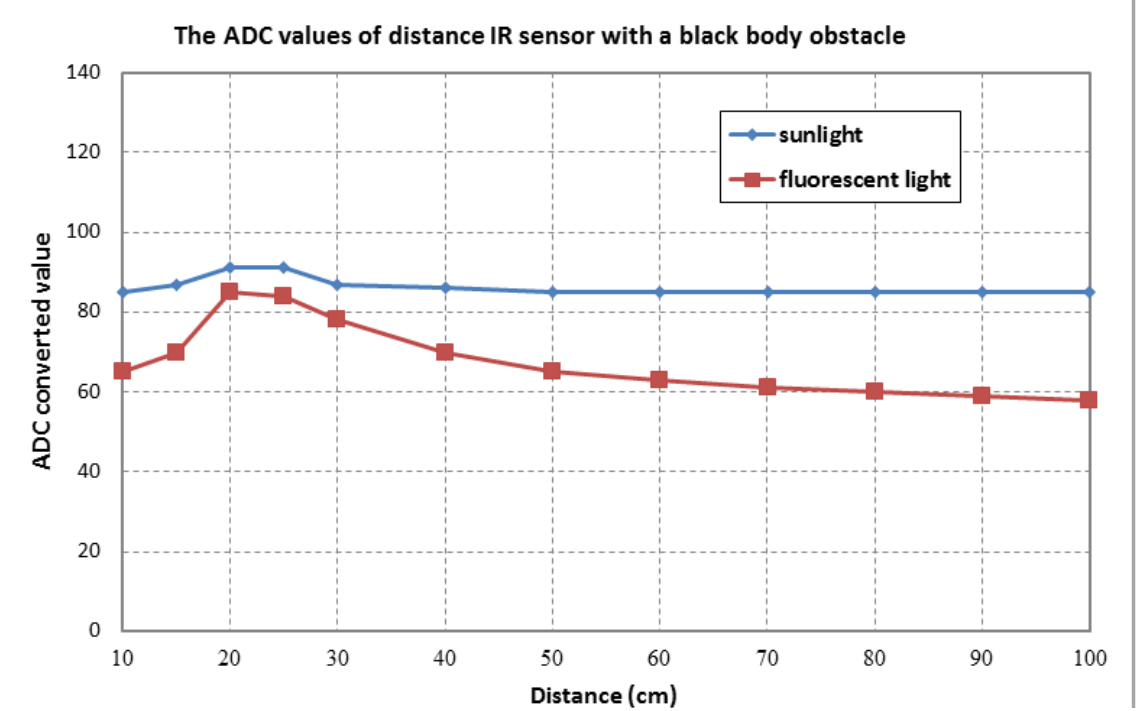

Fig. 9. ADC converted values of distance IR sensors. (a) White body robot. (b) Black body robot.

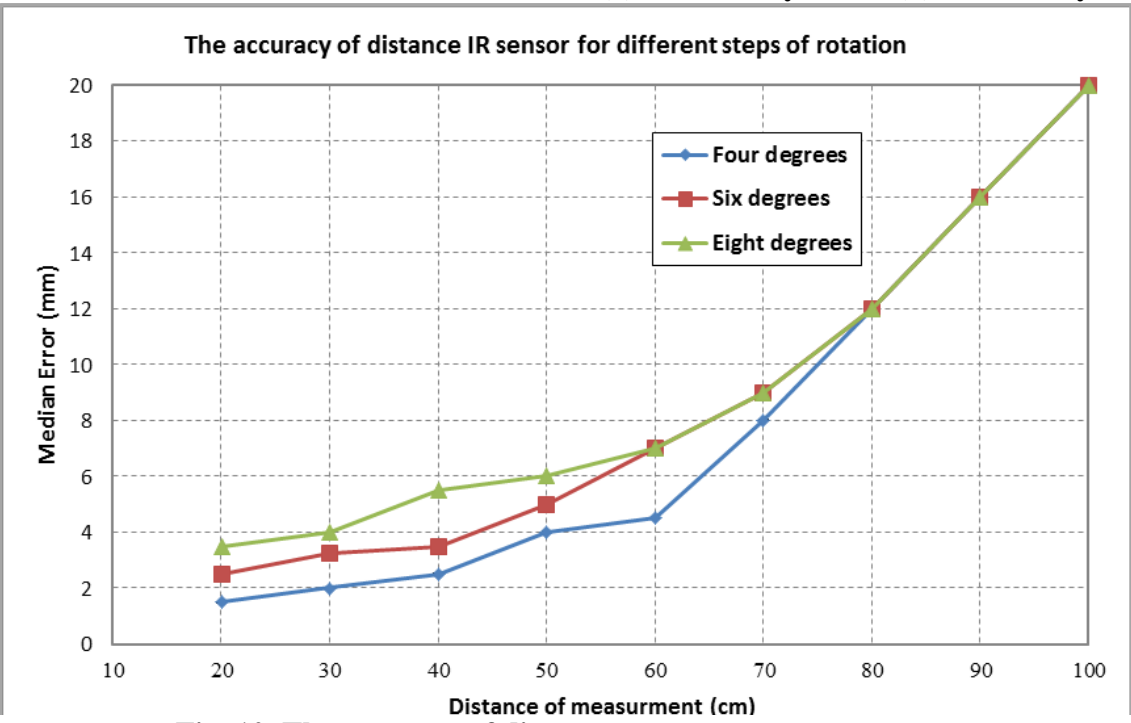

Fig. 10. The accuracy of distance measurement sensor. 


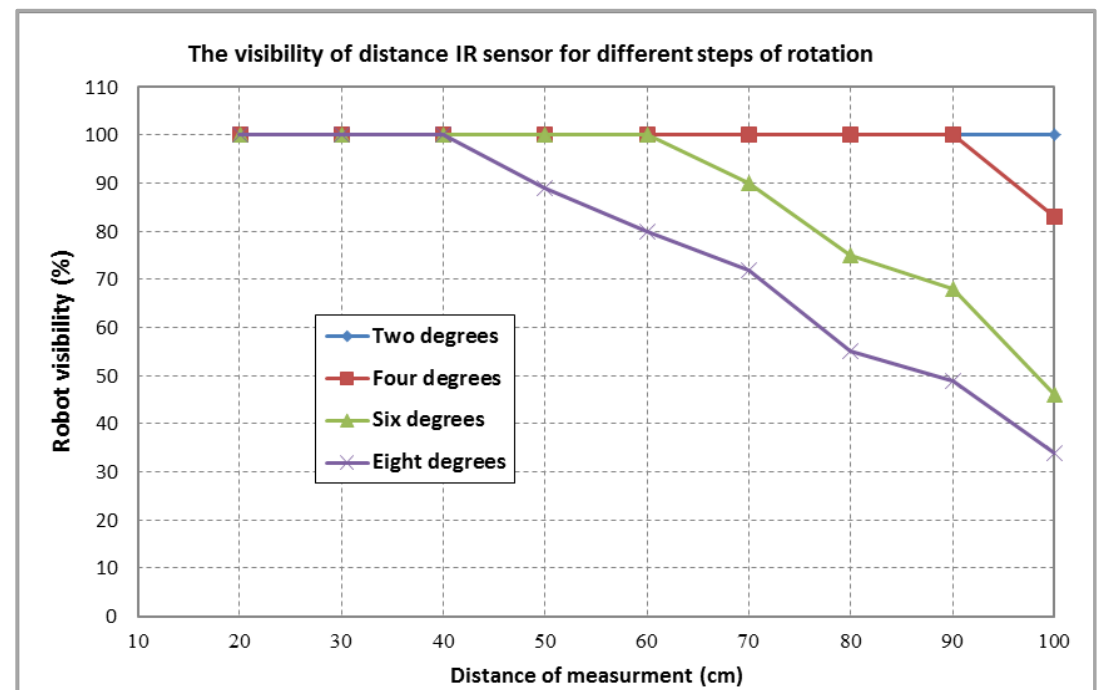

Fig. 11. The visibility of distance measurement sensor.

\section{CONCLUSION}

This paper focused on a study for choosing a suitable environment for measuring the distance between two robots and also, for measuring the communication range between two robots by using low-cost infrared sensors.

The maximum communication range between two robots reach to $23 \mathrm{~cm}$ when both robots have infrared sensors supplied by 5 volts and the value of reference voltage of comparator circuit at each IR detector circuit is 0.7 volts. This maximum communication range can be increased to $72 \mathrm{~cm}$ by equipped each robot with 12 infrared sensors instead of 6 .

The best detection range of distance IR sensor occurs when robots with white bodies in fluorescent light environment are used. The median error of the distance IR sensor reading can be decreased by reducing the rotation angle of this sensor.

\section{REFERENCES}

[1] A. T. Rashid, M. Frasca, A. A. Ali, A. Rizzo, and L. Fortuna "Multi-robot localization and orientation estimation using robotic cluster matching algorithm", Robotics and Autonomous Systems, Vol. 63, pp. 108$121,2015$.

[2] A. T. Rashid, W. H. Zayer, and M. T. Rashid, " Design and Implementation of Locations Matching Algorithm for Multi-Object Recognition and Localization", Iraqi Journal of Electrical and Electronic Engineering, Vol. 14 No. 1, 2018.

[3] Z. Y. Ibrahim , A. T. Rashid, and A. F. Marhoon, " An algorithm for Path planning with polygon obstacles avoidance based on the virtual circle tangents", Iraq J. Electrical and Electronic Engineering, Vol. 12, No. 2, pp. 221-234, 2016.

[4] Z. Y. Ibrahim , A. T. Rashid, and A. F. Marhoon, " Prediction-Based Path Planning with Obstacle Avoidance in Dynamic Target Environment ", Basrah Journal for Engineering Sciences, Vol. 16, No. 2, pp. 48 $-60,2017$.

[5] A. A. Ali, A. T. Rashid, M. Frasca, and L. Fortuna, "An algorithm for multi-robot collision-free navigation based on shortest distance", Robotics and Autonomous Systems, vol. 75, pp. 119-128, 2016.
[6] D. Zook, U. Bonne, and T.Samad, "Sensors in control systems," Control Systems, Robotics, and Automation, vol. XXI, 2000.

[7] F. Wu, and J. Williams, "Design and implementation of a multi-sensor based object detecting and removing autonomous robot exploration system," Journal of Computer and Communications, vol. 2, pp. 8-16, 2014.

[8] S. Rathod, V. Bansal, K. T. Patil, "Real-time speed based obstacle detection with path planning," International Journal of Advance Foundation and Research in Science \& Engineering (IJAFRSE), vol. 2, Issue 10, pp. 32- 41, April 2016.

[9] J. I. Bangash, A. Abdullah, A. Khan, "Issues and challenges in localization of wireless sensor networks," Sci.Int (Lahore), pp. 595-603, 2014.

[10] S. Daud, N. H. Mahmood, P. L. Leow,and F. Kh. C. Harun, "Infrared sensor rig in detecting various object shapes," International Journal of Advanced Research in Electrical, Electronics and Instrumentation Engineering, vol. 2, issue 10, pp. 4726-4732, October 2013.

[11] O. A. Hasan, R. S. Ali, A. T. Rashid, "Centralized approach for multi-node localization and identification", Iraq J. Electrical and Electronic Engineering, vol.12, No 2, pp. 178-187, 2016.

[12] O. A. Hasan, A. T. Rashid, R. S. Ali, "Hybrid approach for multi-node localization and Identification", Basrah Journal for Engineering Sciences, vol.16, No 2, pp. 1120, 2016.

[13] J. Guivant, E. Nebot, and S. Baiker, "Localization and map building using laser range sensors in outdoor applications," Journal of Robotics Systems, vol.17, no.10, pp. 565-583, 2000.

[14] J. Guivant, F. Masson,and E. Nebot, " Simultaneous localization and map building using natural features and absolute information," Robotics and Autonomous Systems vol.40, pp. 79-90, 2002.

[15] O. A. Hasan, A. T. Rashid, R. S. Ali, J. Kosha, "A practical performance analysis of lowcost sensors for indoor localization of multi-node systems", International Conference on Internet 
International Journal of Computer Applications (0975 - 8887)

Volume 182 - No. 26, November- 2018

Technologies \& Applications, pp. 284-287, 2017.

[16] V. Jungnickel, V. Pohl, S. Nönnig, and C. V. Helmolt, "A Physical Model of the Wireless Infrared Communication Channel ", pp.159-209. IEEE Journal
On Selected Areas In Communications, Vol. 20, No. 3, 2002.

[17] Sharp 2Y0A21 distance measurement IR sensor datasheet. 